\title{
Intact spatial updating during locomotion after right posterior parietal lesions
}

\author{
John W. Philbeck ${ }^{\mathrm{a}, *}$, Marlene Behrmann ${ }^{\mathrm{a}}$, Sandra E. Black ${ }^{\mathrm{b}}$, Patricia Ebert $^{\mathrm{b}}$ \\ ${ }^{a}$ Department of Psychology, Carnegie Mellon University, Pittsburgh, PA 15213, USA \\ ${ }^{\mathrm{b}}$ Sunnybrook Health Science Center, Toronto, ON, Canada
}

Received 26 February 1999; received in revised form 23 November 1999; accepted 1 December 1999

\begin{abstract}
One function of the posterior parietal cortex (PPC) is to monitor and integrate sensory signals relating to the current pointing direction of the eyes. We investigated the possibility that the human PPC also contributes to spatial updating during larger-scale behaviors. Two groups of patients with brain injuries either including or excluding the right hemisphere PPC and a group of healthy subjects performed a visually-directed walking task, in which the subject views a target and then attempts to walk to it without vision. All groups walked without vision accurately and precisely to remembered targets up to $6 \mathrm{~m}$ away; the patient groups also performed similarly to the healthy controls when indicating egocentric distances using non-motoric responses. These results indicate that the right PPC is not critically involved in monitoring and integrating non-visual self-motion signals, at least along linear paths. In addition, visual perception of egocentric distance in multi-cue environments is immune to injury of a variety of brain areas. (C) 2000 Elsevier Science Ltd. All rights reserved.
\end{abstract}

Keywords: Egocentric distance perception; Space perception; Perception/action; Path integration; Navigation; Walking

\section{Introduction}

Many human activities require keeping track of, or updating, the current position of the body and its parts. To perceive a stable visual world, for instance, the visual system must take eye and body motions into account so that it does not incorrectly attribute all visual motion on the retina to motion in the external world. A striking example of human spatial updating ability on a larger scale is the accuracy with which the average observer can walk without vision to a previously-viewed target location. After viewing a target in a well-lit environment, the average observer stops very near the target's location when walking without vision, even when the target is up to $20 \mathrm{~m}$ or more away from the walking origin $[11,17,44,51,58,62]$.

\footnotetext{
* Corresponding author. Tel.: +1-412-268-4237; fax: +1-412-2682798 .

E-mail address: philbeck@andrew.cmu.edu (J.W. Philbeck).
}

Because this task is performed without visual feedback, it is known as "visually-directed walking". By contrast, "visually-guided" actions are performed under direct visual control [34].

Good visually-directed walking performance is indeed impressive, given that the observer is prevented from fixing his or her location by referring to visible landmarks. When landmarks are directly perceptible, either through vision or through some other sensory modality, one may determine one's position without reference to internally-generated self-motion information. This form of position-based navigation is known as "piloting" [37]. Without vision, however, one must update one's movement through other sensory signals, such as proprioception (limb position information), kinesthesis (limb movement information), efference copy of the motor commands controlling muscle activation, and vestibular signals. The fact that healthy humans typically perform well despite the absence of vision suggests that they are quite adept at 
using some combination of the remaining forms of information to update their location while walking along linear paths. In this paper, we restrict our attention to non-visual locomotion; this will allow us to exclude piloting as a method for spatial updating and to focus on forms of updating based on non-visual internallygenerated self-motion signals.

Accurate visually-directed walking is also critically dependent upon the visual information that specifies the target's location from the observer's initial viewpoint [35]. Previous work has shown that walking responses are tightly coupled with the perceived distance of the target $[44,45]$. Despite progress in analyzing visually-directed walking into its component subprocesses, however, little is known about the neural substrates of these subprocesses in humans. One approach to studying such issues is to observe the behavior of people with localized brain injuries and to use those data to make inferences about what brain structures underlie normal behavior in healthy humans. In the study described below, we evaluate the performance of brain-injured patients on tests of visual space perception and spatial updating. Our primary interest is in the neural substrates of spatial updating during nonvisual locomotion, but because there has been so little systematic study of egocentric distance perception in brain-injured humans, we will also emphasize this aspect of our work. Visual perception of a target's location is a fundamental input to visually-directed walking, and therefore one must test thoroughly a patient's ability to perceive egocentric distances in order to evaluate the spatial updating component of visually-directed walking. The experiment we report below addresses this issue and in so doing establishes some critical basic findings.

A target's location, seen from an observer's viewpoint, is specified by its visual direction and its egocentric (or absolute) distance. Although considerable work has been devoted to investigating the perception of visual direction in brain-injured patients ${ }^{1}$, our concern here lies more in egocentric distance perception, which has rarely been studied systematically in neurological populations [9]. Early reports were based on

\footnotetext{
${ }^{1}$ Patients with right hemisphere parietal lesions sometimes display symptoms of "hemispatial neglect", in which targets on the left side of the egocentric body midline are apparently mislocalized towards the right or are ignored altogether [5]. The stimuli in these localization studies are often arrayed on a flat piece of paper or a computer monitor. Thus, from the observer's point of view, the stimuli vary primarily in visual direction in the horizontal plane rather than egocentric distance. Sometimes these tasks are loosely called distance perception tasks, perhaps to emphasize the lateral separation of the stimuli within the plane of the testing surface. However, one should not assume that deficits in "distance perception" as revealed by these tasks necessarily generalize to include deficits in egocentric distance perception.
}

informal clinical observations, and in some cases it is possible that the patients' ability to perceive egocentric distances was underestimated because reaching was used as a behavioral response [30,49]. Reaching by itself is not sufficiently diagnostic because visually guided reaches can be impaired even when non-motoric indications of spatial relations remain relatively normal $[42,47]$. However, other informal reports that are not subject to this methodological criticism describe patients who are clearly profoundly impaired in judging spatial relations. In "visual disorientation", for example, patients have difficulty discriminating between the egocentric distances of two points widely separated in depth, and some have been observed to collide with objects while walking about, even though they can apparently see and recognize those objects [12]. There is often bilateral parietal damage in cases of visual disorientation ([12,31]; see also [49]).

One type of spatial updating performed during visually-directed walking that is particularly important involves updating a representation of one's own location during the walk. This process is referred to as path integration or dead reckoning $[20,37,39]$. In it, one fixes one's current position with respect to the starting position by updating an estimate of one's current velocity and/or acceleration. This self-motion information could come from a variety of sources, such as kinesthesis, the vestibular apparatus, or efference copy of motor commands. Integrating velocity (or doubly integrating acceleration) over time allows the organism to update its change in position relative to the starting position. This change in position provides a minimal representation of both the location of the origin and the organism's spatial relationship to it. Aside from these minimal representations, however, path integration may be accomplished in the absence of a specified destination or indeed any other representation of locations in the immediate environment. Other updating processes could also play a role, such as updating the remembered location of a destination or other environmental features $[7,35,50]$.

The human posterior parietal cortex (PPC) is strongly implicated in at least one type of spatial updating. In particular, it has been found to play a crucial role in updating extra-retinal signals related to eye position [16,29]. This evidence comes from a double-step saccade paradigm, in which two visual targets are flashed in sequence in an otherwise dark room [26]. The task is to fixate the remembered target locations in the order of presentation. In a critical manipulation, the targets are flashed so briefly that saccades cannot begin until after both targets have been extinguished; thus, after the first saccade lands, there is a discrepancy between the head-centered coordinates of the second target location and the retinotopic coordinates. In order to accurately execute the 
second saccade, the visuomotor system must take into account the change in eye position that occurs during the first saccade, and because the saccades are made in complete darkness, this eye-position sensing must be mediated by extra-retinal information. When patients with unilateral PPC damage attempt to perform this task, their saccades to the second target are impaired when the first saccade causes their eye to point into the contralesional half of visual space $[16,29]$. Thus, PPC lesions are associated with deficient eye position updating. Consistent with this lesion site, physiological studies in the monkey implicate neurons in the lateral intraparietal area (LIP) in eye position monitoring $[2,24]$.

There is evidence that the PPC's role in updating motor signals extends beyond the extra-ocular muscles to include other muscles groups, but the limits of the generality of this updating function remain unknown. Neuropsychological work and transcranial magnetic stimulation studies suggest that the PPC functions to maintain an updated estimate of the current position of the arms [15,65]. In both monkeys and humans, lesions to certain regions of the PPC can produce a disorder of reaching behavior, known in humans as optic ataxia $[19,27,32,42]$. However, this deficit is not the result of a general impairment in updating limb position, because the patients can accurately reach without vision to parts of their body indicated by touch [42]. Taken together, these findings suggest that the portions of the PPC involved in updating may be quite localized and perhaps anatomically segregated according to the effector systems they monitor.

Evidence consistent with a parietal lobe role in monitoring the locomotor effector system does exist, but important details have yet to be established. PPCinjured individuals have been found to perform poorly on some tasks involving locomotion $[4,28,55]$, but it is difficult to ascertain whether updating is the primary source of the impairment. The tasks in these studies not only provided some degree of visual guidance, but also involved complex paths that confounded linear translations and rotations. It is entirely possible that brain injuries might impair updating during one of these types of motions without affecting updating during the other, but few studies have addressed this issue.

Another hint that the PPC may be involved in spatial updating during locomotion is that PPC-injured individuals have been found to be deficient at tasks requiring mental transformations of the body in space [57]. The PPC is also active when healthy humans perform tasks involving mental transformations of certain body parts $[6,67]$. It is possible that a similar process underlies self-motion monitoring, and if so, one might expect damage to the PPC to result in generalized impairments in updating self-motion. However, the possible connection between mental transformations of body parts and self-motion monitoring has not been firmly established.

Our primary goal here was to explore the neural substrates of the spatial updating underlying visuallydirected walking, with particular emphasis placed on the possible role of the PPC. The possible lateralization of this updating function has not been studied in detail, but there is some evidence that deficits in updating eye position may be more pronounced after right PPC damage [29]; the right hemisphere dominates in other forms of spatial processing as well (for a review, see [14]). As a first step toward addressing the possible laterality of self-location updating, we tested the hypothesis that the right PPC plays a crucial role in this function. In addition, to shed some light on the possible differential effects of brain damage on updating during translational versus rotational whole-body motions, we further restricted our focus to updating during walks along linear trajectories.

We asked three groups of participants to perform the visually-directed walking task: two groups of brain-injured patients, with injuries either including or excluding the right PPC, and one healthy control group. In visually-directed walking, the subject views a target and then attempts to walk to its location without vision. To further explore the spatial updating performed during walking, we also tested updating under conditions in which no target was specified before walking began. For this, we used an "experimenterguided walking" task, in which subjects estimated distances walked without vision while guided along a straight path by an experimenter [33,36,54]. As mentioned above, a crucial step for interpreting the effects of these manipulations is to rule out impairments in the ability to visually perceive the target's initial location. We did this by asking subjects to indicate the distance to targets using verbal distance estimation and distance matching, two methods that do not rely upon spatial updating.

\section{Method}

\subsection{Participants}

Three groups of participants consented to take part in this study. (1) Participants in the healthy control group $(N=12$ : seven females, five males; mean age: 68 years) had no history of neurological disease. (2) Patients in the brain-injured control group $(N=7$ : one female, six males; mean age: 41 years) had brain injuries of mixed etiologies. Although one patient's lesion included a very small portion of the right hemisphere parietal lobe (less than $10 \%$ ), this region was otherwise entirely spared in the remaining six patients in this 
group. Three patients in this group were visually agnosic and had right hemisphere occipito-temporal lesions; two others demonstrated pure alexia and had circumscribed left occipital lesions. A sixth patient (JM) had a right fronto-temporal lesion with some involvement of the thalamus and basal ganglia. The seventh patient had a large right fronto-temporal lesion resulting from a craniotomy. Three patients in this group (including JM) were mildly hemiplegic at the time of testing, but could walk with the assistance of a cane; the rest had no obvious walking impairments. Four of these patients have been described in more detail elsewhere $[3,21,38,63]$. (3) Patients in the parietal group $(N=6$, all males; mean age: 67 years) were selected for inclusion in this group by the presence of a cerebral vascular accident involving the middle cerebral artery. With one exception, each had brain injuries confined to the right hemisphere with some involvement of the parietal lobe. One patient, in addition to his right hemisphere damage, had a small (3 cc) lesion in the left putamen. Three patients in this group ordinarily walked with a cane due to mild left-sided hemiplegia, while one other had less severe hemiplegia at the time of testing and walked well without assistance. The last two reported some temporary left hemiplegia following their stroke, but this had resolved by the time of testing. An additional patient was excluded from the analysis because in one experimental block she appeared to be indicating the distance of one of the experimenters, rather than the nominal target. Demographic details and lesion information of the two patient groups are reported in Table 1 and neuroima- ging data for five of the six patients in the parietal group are shown in Fig. 1. Fig. 2 shows neuroimaging data for patient JM in the brain-injured control group, who, as we will see, was the only patient to manifest deficits on any of the tests we conducted.

All participants were right handed, except for one patient in the brain-injured control group; each had visual acuity of at least $20 / 40$, corrected if necessary, and they all reported having normal color vision. Neither optic nor motor ataxia was evident upon clinical observation of the patients. Patients were medically stable and ambulatory at the time of testing. Lesion sites and volumes were verified by CT scans.

Participants in all groups were screened for hemispatial neglect using the conventional sub-test of the Behavioral Inattention Test [64], which includes line bisection, line cancellation, letter cancellation, figure copying, and figure drawing tasks. The cut-off score for normal performance is 129 out of a total of 146 points; four patients in the parietal group scored between 103 and 120, indicating mild hemispatial neglect, and the rest scored within normal limits.

\subsection{Apparatus}

\subsubsection{Laboratories}

The experiment took place in a well-lit indoor gymnasium $(33 \times 13 \mathrm{~m})$. Four participants in the parietal group were tested in a different room $(18 \times 11 \mathrm{~m})$ in another city. Stimulus locations were marked on the floor with transparent tape and were not visible from the observation location.

Table 1

Demographic, lesion and neglect details for patients in the parietal and brain-injured control groups ${ }^{\mathrm{a}}$

\begin{tabular}{|c|c|c|c|c|c|c|c|c|c|}
\hline Patient & Group & Sex & Age & Hand $^{\mathrm{b}}$ & Time test $\mathrm{t}^{\mathrm{c}}$ & Volume $^{\mathrm{d}}$ & Neg. score ${ }^{e}$ & 39 involve $^{\mathrm{f}}$ & 40 involve $^{f}$ \\
\hline JB & Parietal & Male & 68 & Right & 41 & 123 & 103 & $50-89 \%$ & $>90 \%$ \\
\hline RRB & Parietal & Male & 65 & Right & 57 & 90 & 108 & $<10 \%$ & $>90 \%$ \\
\hline KW & Parietal & Male & 77 & Right & 5 & 186 & 120 & $50-89 \%$ & $<10 \%$ \\
\hline $\mathrm{RB}$ & Parietal & Male & 79 & Right & 4 & 19 & 114 & $10-49 \%$ & $<10 \%$ \\
\hline $\mathrm{AA}$ & Parietal & Male & 59 & Right & 16 & 138 & 131 & $50-89 \%$ & $50-89 \%$ \\
\hline ST & Parietal & Male & 53 & Right & 11 & 31 & 143 & $10-49 \%$ & $10-49 \%$ \\
\hline JW & Control & Male & 41 & Left & 37 & & $\mathrm{~g}$ & none & none \\
\hline EL & Control & Female & 49 & Right & 24 & & 143 & none & none \\
\hline SM & Control & Male & 24 & Right & 41 & & 144 & none & none \\
\hline DK & Control & Male & 68 & Right & 68 & & 143 & none & none \\
\hline JS & Control & Male & 26 & Right & 69 & & 141 & none & none \\
\hline JM & Control & Male & 54 & Right & 72 & 126 & 139 & none & none \\
\hline $\mathrm{RD}$ & Control & Male & 24 & Right & 31 & 252 & 146 & none & $<10$ \\
\hline
\end{tabular}

\footnotetext{
${ }^{a}$ Note: Except for JM and RD, lesion volumes were not calculated for the brain-injured control group.

${ }^{\mathrm{b}}$ Preferred hand.

${ }^{\mathrm{c}}$ Time of testing, post-onset (months).

${ }^{\mathrm{d}}$ Total lesion volume (cc).

${ }^{\mathrm{e}}$ Neglect score (maximum score $=146$ ). Bold indicates below-normal performance.

${ }^{\mathrm{f}}$ Percentage of Brodmann parietal areas 39 and 40 involved in the lesion. Only JBs lesion involved Brodmann parietal areas 5 and 7.

${ }^{\mathrm{g}}$ Not measured; JW is likely to perform poorly on standard neglect tests because of his perceptual impairments, but this poor performance would not be differentiated by side (left or right).
} 


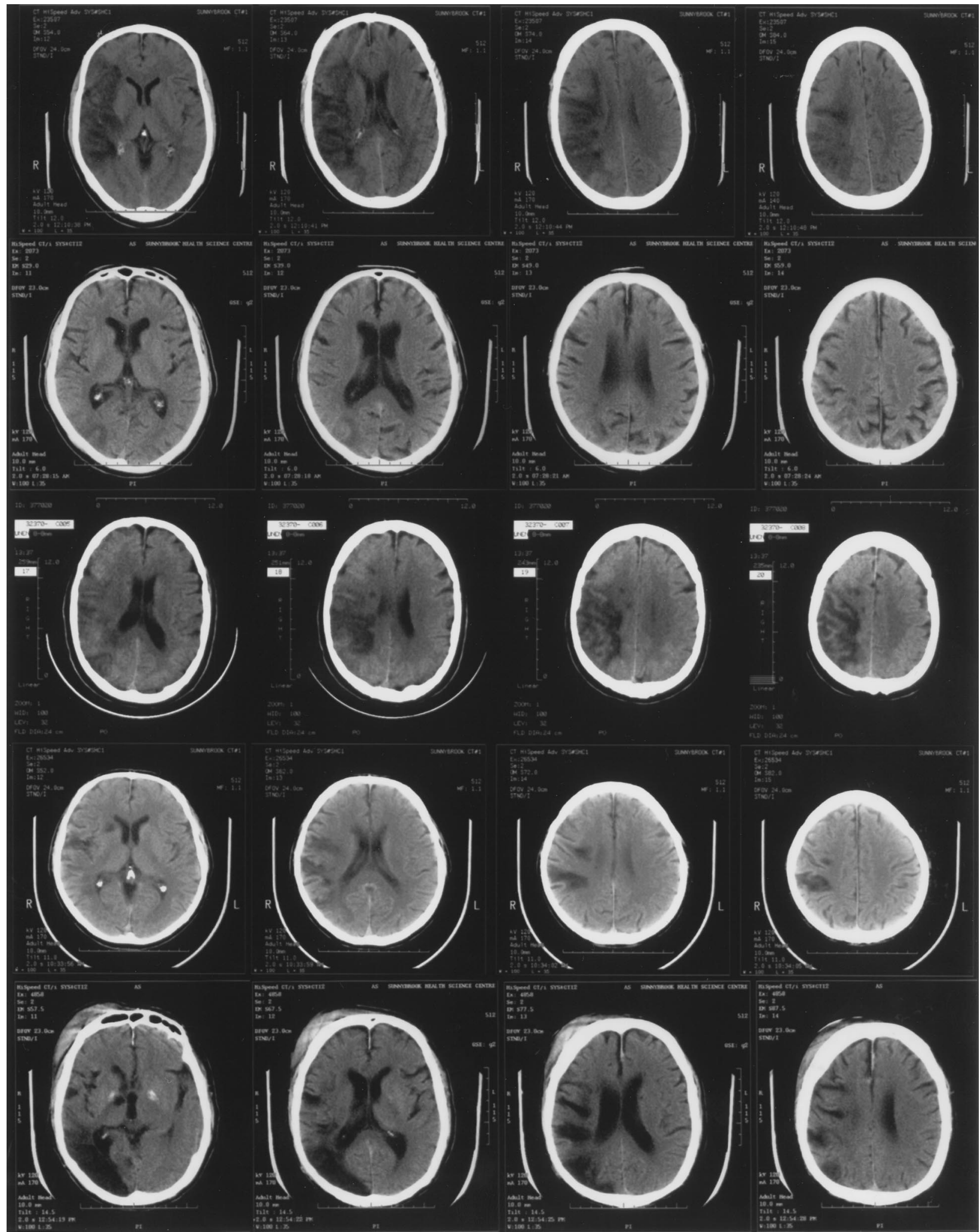

Fig. 1. Neuroimaging data for five of the six patients in the parietal group; the images were obtained by computerized tomography. Images of patient RRB were not available for publication. Each row of images is from the same patient, and the sequence of patients follows the same order as that in Table 1. From top to bottom: AA, RB, JB, ST, and KW. Radiological coordinates are used, with the right hemisphere appearing on the left side of the images and the anterior of the brain appearing on the top. 


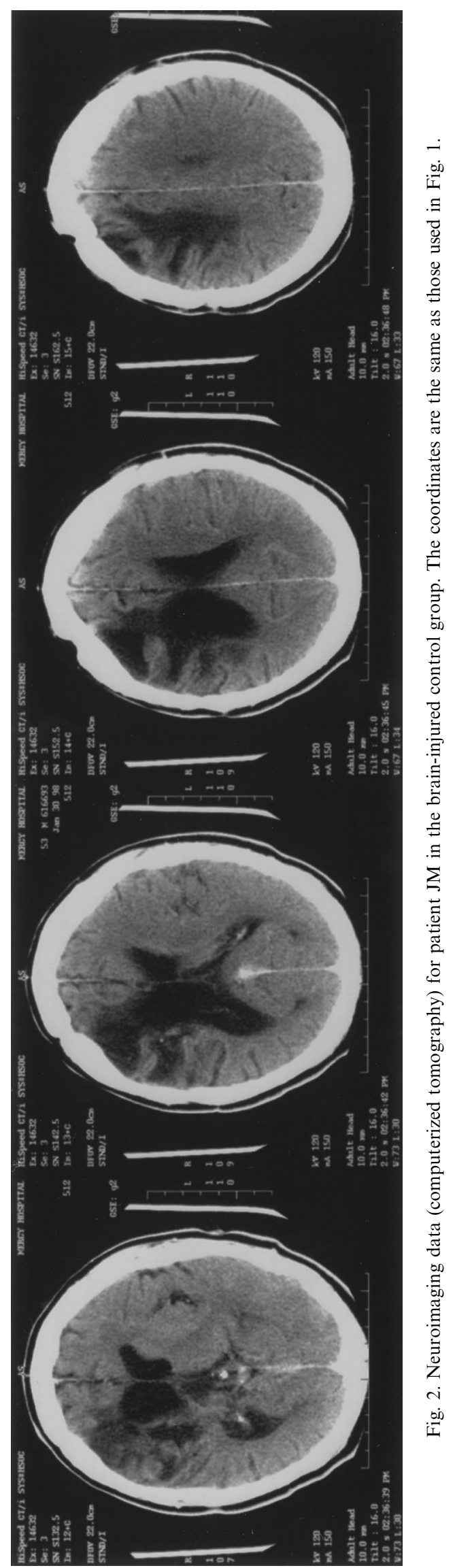

\subsubsection{Stimuli}

The visual target was a small rectangular or spherical object (less than $13 \mathrm{~cm}$ square). Because we thought deficits in the parietal patients might be more pronounced on the contralesional side, we presented the targets equally often at $45^{\circ}$ to the left or right of the egocentric body midline. During the visual phase of each trial, viewing was binocular and head motion was unconstrained. Some individuals in the parietal group demonstrated mild hemispatial neglect and might potentially have had difficulties detecting targets on the contralesional side. Measuring the impact of neglect on target detection was not a goal in the current experiment, so we verified that all subjects had detected the target by having them report the target's color before attempting to walk to it; the target color on these trials was randomly chosen out of five possibilities. Participants were instructed to base their responses on the physical distance of the target, as measured from the tip of the toes to the front surface of the target.

\subsection{Design}

Each subject participated in five treatment conditions, which consisted of various combinations of stimulus and response modalities: vision/verbal, vision/ match, vision/walk, proprioception/verbal, and proprioception/walk. During visual input trials, the subject viewed a target, then indicated its distance either by verbal estimation, by picking out a location marker with a matching distance, or by attempting to walk to the target without vision (visually-directed walking). During proprioception input trials (experimenterguided walking), the stimulus distance was specified by guiding the blindfolded observer along a straight path of a certain length. The "proprioception" label emphasizes that the stimulus distance was specified non-visually, but other forms of self-motion information may also have contributed during these trials. After being guided along the stimulus path, the subject then gave a verbal estimate of that path length and attempted to reproduce the path length by walking without vision.

In addition to the between-groups "neurological status" factor (healthy control, brain-injured control, and parietal) and the five within-subject treatment conditions, stimulus orientation $\left(45^{\circ}\right.$ left $/ 45^{\circ}$ right $)$ and stimulus distance $(2.5 \mathrm{~m} / 5 \mathrm{~m})$ were varied within subjects. Each combination of the within-subject factors was measured three times. Four other stimulus distances $(2,3,4$, and $6 \mathrm{~m})$ were tested once apiece on the left and right in all five treatment conditions. This permitted an increased range and density of distances sampled while keeping the number of trials manageably small.

The experiment was conducted in three blocks, such 
that the two visual-input conditions were blocked together (vision/verbal and vision/match) and the two proprioception-input trials were blocked together (proprioception/verbal and proprioception/walk). Vision/ walking trials were conducted in a separate block. Trials within each block were presented in a pseudorandom order, such that the first two trials in the block used one of the four additional stimulus distances. The block order was counterbalanced for the healthy control group, with two participants randomly assigned to each of the six possible orders of the three blocks. This counterbalancing was not strictly possible for the other two participant groups, as there were fewer participants in these groups, but subjects were randomly assigned to participate in different block orders to the extent possible.

\subsection{Procedure}

\subsubsection{Verbal and matching responses}

While facing straight ahead, subjects viewed the target and gave a verbal estimate of its distance, using their choice of unit. The experimenter then guided them in a rotation of $180^{\circ}$ (randomly to the left or right), until they faced a strip of white paper $0.61 \mathrm{~m}$ wide and about $8 \mathrm{~m}$ long. On the strip were $1611 \times$ $11 \mathrm{~cm}$ squares of colored paper, spaced in $0.36 \mathrm{~m}$ increments. Each square had a different common color name ("pink", "dark blue", etc.) The task was to give the color name of the patch whose distance matched that of the target seen before the $180^{\circ}$ rotation.

\subsubsection{Visually-directed walking}

Subjects viewed a target, reported its color, then covered the eyes with a blindfold and attempt to walk to the target location. All subjects held onto the upper arm of the experimenter for support during walking. The experimenter remained blind to the target distance on each trial by closing his eyes until an assistant removed the target immediately before walking began. Subvocal pace counting was discouraged by requiring subjects to repeat aloud a nonsense phrase. In addition, on each trial the experimenter walked at one of three randomly selected speeds: slow, medium, or fast (approximately 1.0, 1.5, and $2.0 \mathrm{~m} / \mathrm{s}$, respectively). Because the subjects did not know which speed would be used until after vision of the target was obscured, pace counting would be an unreliable strategy. Subjects remained blindfolded during the outbound and return paths and did not receive any error feedback.

\subsubsection{Experimenter-guided walking}

Each of these trials was conducted in three phases - a stimulus phase followed by two responses phases (verbal and walked reproduction), each of which was based on the same stimulus phase. Before each trial, subjects were oriented such that the walking path was approximately $45^{\circ}$ to the right or left and allowed to raise the blindfold to view the workspace. The walking path was denoted by a tape measure that stretched out from the starting position to a distance of $18.3 \mathrm{~m}$. After viewing the workspace for several seconds, subjects lowered the blindfold and were oriented in the direction of the walking path. Thus, walking was always performed in the same direction, but during the preview of the workspace before walking, the path lay either on the subjects' left or right. We did this because we thought that, as is the case in updating eye movements [16,29], parietal patients may update more poorly in regions of space that lay on their contralesional side during the visual preview. During the stimulus phase, all subjects grasped the experiment's upper arm as he walked along a straight path between 2 and $6 \mathrm{~m}$ long. After the experimenter stopped, subjects verbally estimated the distance walked (verbal response phase). After giving that verbal estimate, subjects then attempted to reproduce the distance walked, again by walking blindfolded (walked reproduction phase). The starting point of the reproduction phase began at the terminal location of the stimulus phase, and the stopping point of the reproduction phase was determined by the subject. During the reproduction phase, subjects continued walking in the initial direction while holding onto the experimenter's arm. The subjects repeated aloud a different nonsense phrase during the stimulus and the reproduction phases, and again, the experimenter walked at one of three randomly selected speeds. At the end of the reproduction phase, the subjects were led back to the starting location, still without vision.

\subsection{Data analysis}

Constraints on subject availability did not allow us to select a healthy control group that was strictly sexmatched with the parietal group, so we first compared the responses of the seven women and five men in the healthy control group. There were no main effects or interactions involving the gender variable revealed by an analysis of variance (ANOVA) of the raw data, so we ignored gender in subsequent analyses.

In analyzing the data, we looked for differences in within-subject variability as well as in the mean responses between subjects. To test for mean differences, we used ANOVA to analyze the signed error scores from the responses to the 2.5 and $5 \mathrm{~m}$ stimulus distances, with stimulus side (left or right) included as a variable. Using these responses allowed us to take advantage of the multiple measurements per condition obtained for each subject at these distances. We analyzed variability by finding the straight line that best fit the relation between the physical and indicated dis- 
tances for each subject; we then calculated standard errors of estimate (SEE) for each subject, one for each condition (e.g., "vision/verbal"), and analyzed these SEEs in an ANOVA. For this analysis, we used data from all stimulus distances to take advantage of the increased range of distances. In addition, we pooled the data across stimulus side because the analyses of the mean responses showed there to be neither a main effect of side nor a group $\mathrm{x}$ side interaction, as we report below.

We grouped the data into two sets. The first set contained the verbal and matching indications of the distance of visual targets. This allowed us to compare visual space perception between groups using two different responses that did not require spatial updating. The other set addressed the issue of spatial updating, and compared verbal distance estimates and walking as two response modalities crossed with vision and proprioception as two stimulus modalities. This yielded four conditions (vision/verbal, vision/walk, proprioception/verbal, proprioception/walk) in a $2 \times 2$ design. For each of these two sets of data (distance perception and spatial updating) we performed one ANOVA to test mean differences and another to assess within-subject variability (SEE).

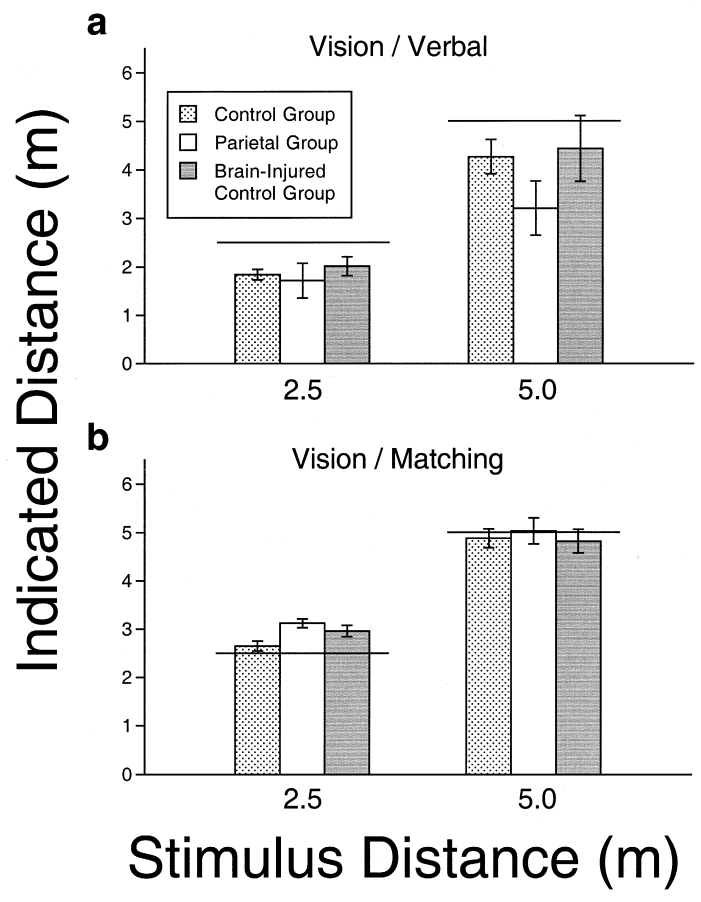

Fig. 3. Average indicated distances of visual targets, for the healthy control group, the parietal group and the brain-injured control group. Verbal estimates and matching responses are shown in panels a and b, respectively. Data have been collapsed over side of presentation (left or right of egocentric body midline). The data from stimulus distances of 2.5 and $5 \mathrm{~m}$ are shown. Error bars denote \pm 1 one standard error of the mean; solid horizontal lines indicate the physical stimulus distance in each condition.

\section{Results}

\subsection{Visual space perception}

There was no overall effect of group $(F(2,22)=0.36$, $p>0.05)$ in the two non-motoric measures of visual space perception. Fig. 3 shows the average verbal and matching responses to the 2.5 and $5 \mathrm{~m}$ targets. There was a tendency toward underestimation in verbal distance judgments, by about $20 \%$ of the physical target distance. Matching responses were more accurate and indicated only a slight overestimation of about $6 \%$. This difference in response modes was reliable $(F(1,22)=28.35, p<0.0001)$. Significant response mode differences are a common finding in space perception studies and are often attributed to differences in the cognitive processes used to generate the responses (e.g., memory of the length of the unit used to describe the observed distance) [13,23]. For our purposes, however, the critical question was whether the response modes differed among groups: they did not $(F(2,22)=2.41, p>0.05)$. Although there was a tendency for the parietal group to verbally underestimate larger distances more than did the other groups, this interaction did not reach significance $(F(2,22)=1.94, p$ $>0.05$ ). Similarly, there was neither a main effect of the side of stimulus presentation nor a group $\times$ side interaction $(F(1,22)=3.54$ and $F(2,22)=0.75$, respectively, both $p \mathrm{~s}>0.05)$. Collapsing across response modality, the groups did not differ in the precision of responses as measured by the standard errors of estimate $(F(2,22)=0.48, p>0.05)$. However, the precision with which the groups used each response type did differ $(F(2,22)=3.60, p<0.05)$. Tukey post-hoc analyses ( $p=0.05)$ confirmed that the parietal group's matching responses were more variable than those of the other two groups. This was primarily due to one patient's matching responses. His verbal and walking responses were much more consistent, and an analysis of his matching errors indicated that he may have been confusing color names (e.g., he gave a response of "gray" when the correct response was "light blue"). These errors, however, were not representative of the group as a whole.

\subsection{Spatial updating during locomotion}

As mentioned above, spatial updating was assessed in ANOVAs on the signed error scores and SEEs, with stimulus mode (vision/proprioception) crossed with response mode (verbal/walking). The groups did not differ significantly across the four treatment conditions in these analyses (Fig. 4). The analyses of signed error scores showed no main effect of group $(F(2,22)=0.56$, $p>0.05)$. Walked responses were generally numerically larger than verbal responses $(F(1,22)=39.41, p<$ 
0.0001); direct comparisons between these two response types in past work has shown them to be numerically quite similar [44], so it is possible that in the current study the walking speed manipulation and/or the requirement of holding onto another person while walking systematically influenced the walked responses somewhat. However, the response mode $\times$ group comparison, which was more critical for our purposes, was not significant $(F(2,22)=2.95, p>0.05)$. All participants performed well when attempting to walk without vision to a previously-viewed target, although there was a tendency toward overwalking - up to $10 \%$ of the physical target distance in the case of the control group (Fig. 4b). Responses under proprioceptive input (experimenter-guided walking) were about $8 \%$ smaller than those for visual targets $(F(1,22)=6.01, p<0.05)$. Underestimation of proprioceptively specified distances is not uncommon [36]. Again, however, this did not differ between groups $(F(2,22)=2.41, p>0.05)$. There was no interaction of group $\times$ stimulus mode $\times$ response mode $(F(2,22)=1.12, p>0.05)$.

Analysis of the variability (SEE) generally showed a similar lack of differentiation by group (group: $F(2,22)=1.63$; group $\times$ stimulus mode: $F(2,22)=1.26$; group $\times$ stimulus mode $\times$ response mode: $F(2,22)=$
0.24 ; all $p \mathrm{~s}>0.05$ ). There was, however, a general increase in variability under proprioceptive input $(F(1,22)=11.19, p<0.01)$, as well as a group $\times$ response mode interaction $(F(2,22)=3.73 ; p<0.05)$. Tukey post-hoc tests $(p=0.05)$ indicated that although the parietal and healthy control groups performed the same in terms of variability, the braininjured control group's verbal responses were somewhat more variable. This increased variability is attributable to patient $\mathrm{JM}$ in the brain-injured control group, whom we will examine in more detail below.

Several interactions involving group were statistically reliable. When collapsing across the four combinations of stimulus and response modalities in the error scores, the groups differed in their responses to targets at different distances $(F(2,22)=9.07, p<$ $0.001)$. This was further qualified by a group $\times$ stimulus mode $\times$ response mode $\times$ distance interaction $(F(2,22)=3.69, p<0.05)$. When verbally estimating the distance of visual targets, the parietal group underestimated far distances more than did the other two groups (see Fig. 4a). Two of the parietal patients consistently reported the distance of the $5 \mathrm{~m}$ target as " 5 feet" (about $1.5 \mathrm{~m}$ ). Because their visual matches and visually-directed walking responses did not reflect this a

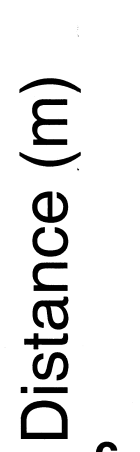

C

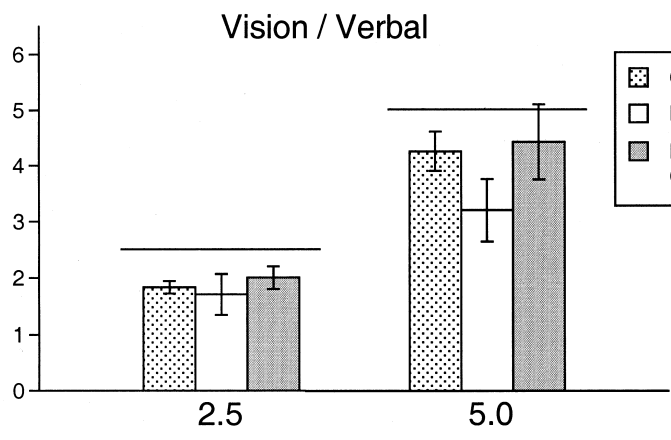

Proprioception / Verbal

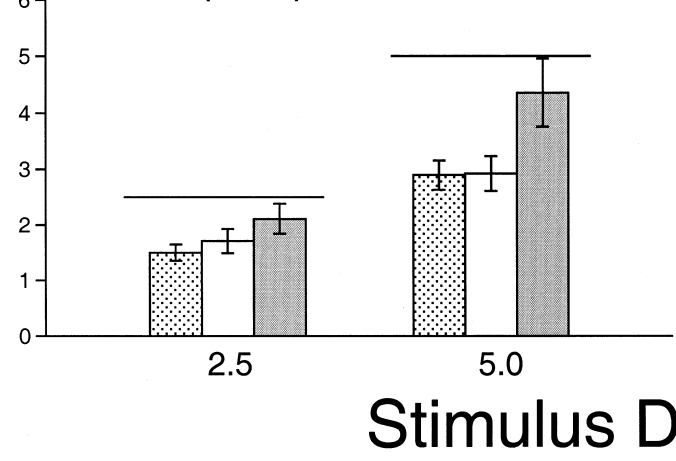

b

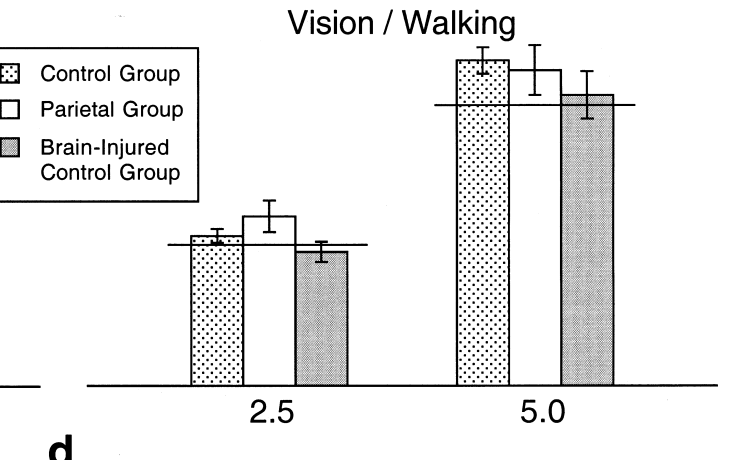

Proprioception / Walking

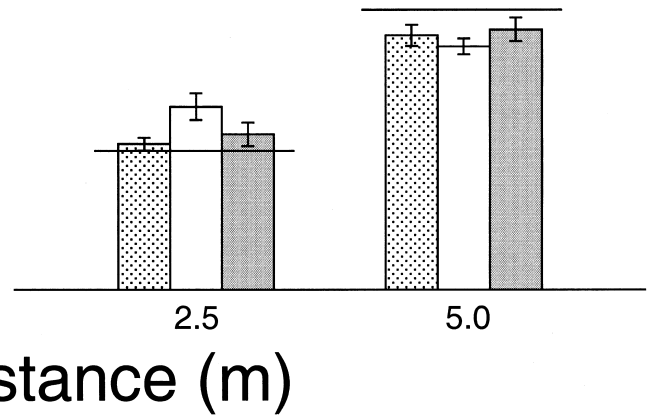

Fig. 4. Average indicated distances for the three subject groups; data from the four combinations of stimulus input modality (vision or proprioception) and response modality (verbal distance estimation or walked reproduction) are shown. Vision/verbal data are repeated from Fig. 3; data have again been collapsed over side of presentation. The data from stimulus distances of 2.5 and $5 \mathrm{~m}$ are shown. Error bars denote \pm 1 standard error of the mean; solid horizontal lines indicate the physical stimulus distance. 
underestimation, we suspect that these patients may have been inconsistent in their choice of units when giving verbal responses, perhaps using yards instead of feet for the far target distances.

Although the parietal and the brain-injured control groups on the whole performed similarly to the healthy participants, one patient in the brain-injured control group (JM) showed clear deficits in trials in experimenter-guided tasks in which the stimulus distances were presented through proprioception. These deficits manifested themselves as a greatly inflated variability under proprioceptive input than under visual input. To illustrate this, we calculated for each subject the difference between the SEEs in "vision" and "proprioception" stimulus conditions. This is analogous to calculating the SEEs differences between the conditions represented in Fig. 4c and $4 \mathrm{a}$ for verbal responses and between Fig. $4 \mathrm{~d}$ and $4 \mathrm{~b}$ for walking. The resulting SEE differences for verbal and walking responses are shown in Fig. 5. JM walked accurately and precisely without vision to previously-viewed targets, but if asked to estimate distances walked without vision while guided by the experimenter, his verbal estimates and walked reproductions became quite erratic. JM's verbal and walking SEE difference scores (circled points in Fig. 5) fell 2.31 and 2.86 standard deviations, respectively, above the mean of the control group. Thus, JM's performance falls unmistakably outside the range of the other subjects. Fig. 6a and 6b show JM's walking performance on visual and proprioceptive input trials; note that Fig. 6a corresponds with the conditions in Fig. 4b, and Fig. 6b with those in

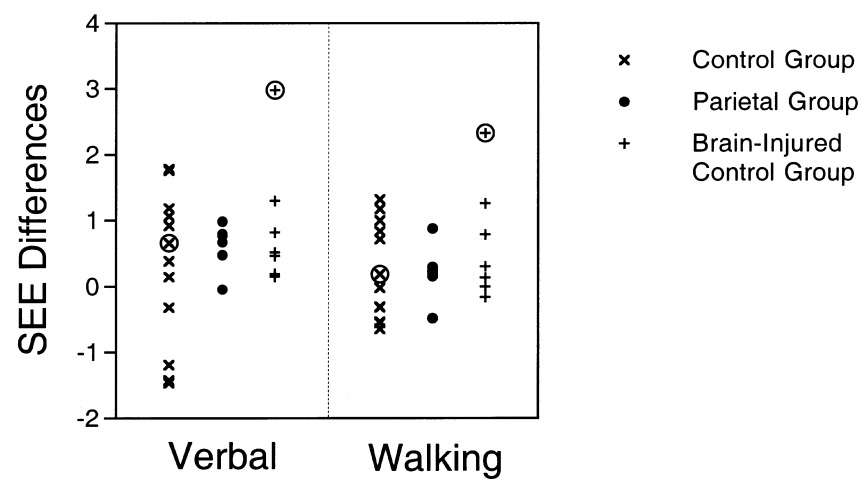

Fig. 5. Standard error of estimate (SEE) differences of the verbal and walking responses of all subjects in the study. To obtain these difference scores, SEEs were first computed as described in Section 2.5; then, difference scores were obtained by subtracting the verbal and walking SEEs under the "vision" conditions from those under the "proprioception" conditions. Thus, the preponderance of positive values here indicates that response variability (as measured by SEE) tended to be greater when stimulus distances were specified by proprioception than by vision. The circled data points indicate SEE differences for subject $\mathrm{C} 3$ in the healthy control group (x symbols) and subject JM in the brain-injured control group (plus symbols).
Fig. 4d. For comparison, the corresponding data of a representative subject in the healthy control group are shown in Fig. 6c and 6d.

\section{Discussion}

Two clear findings emerged from this experiment. First, none of the patients with cortical lesions showed any evidence of impairment in the visual perception of egocentric distance, even though some of them exhibited mild hemispatial neglect or more severe deficits that precluded the visual recognition of faces or other objects. Good performance was manifested in both motoric and non-motoric responses. Thus, visual perception of egocentric distance, at least under conditions which afford many sources of distance information, is immune to injury of a variety of brain areas. The good performance of the parietal group is especially noteworthy, because there is ample evidence that the PPC is responsible for encoding at least some aspects of egocentric space $[10,14,59]$. Our results con-

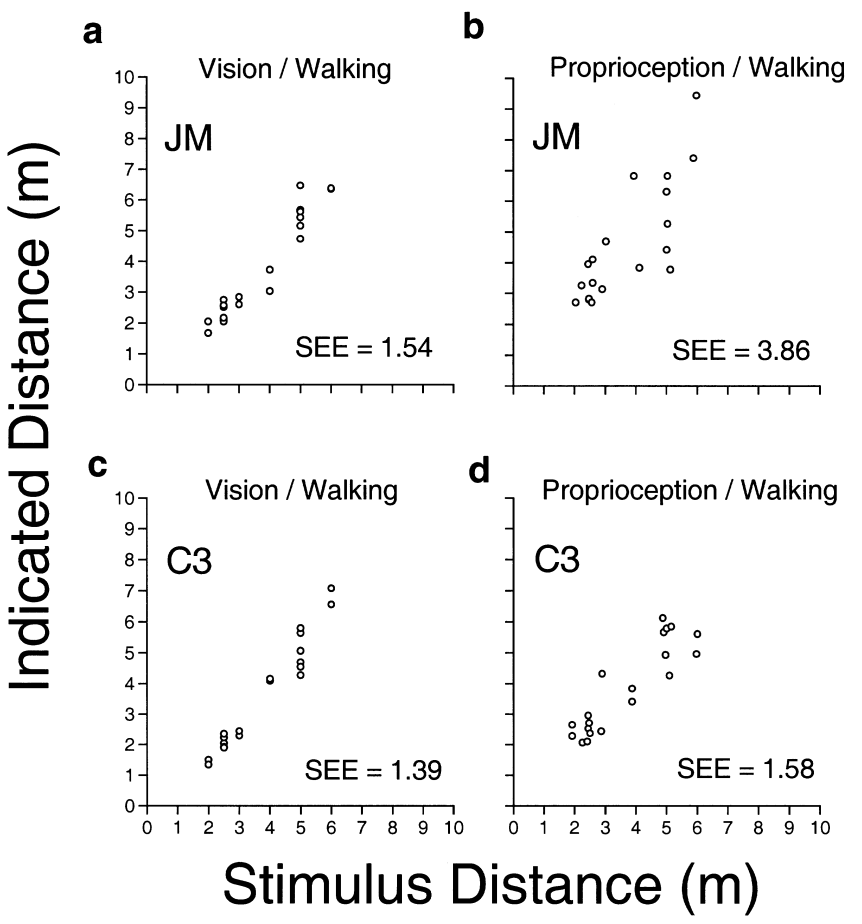

Fig. 6. Distances indicated by blindfolded walking. a and b, Data from subject JM, in the brain-injured control group; c and d, data from subject $\mathrm{C} 3$ in the healthy control group. The standard error of estimate (SEE) of the best fitting line through the data in each panel is also noted. Unlike the other participants in this study, JM's performance was precise when a visual target was specified before walking but became dramatically more erratic when no target was specified beforehand. Subject C3 was chosen as a representative of the healthy control group because C3's SEEs, across walking and verbal responses (not shown), lay at approximately the median of those of the control group (see also Fig. 5). 
firm that the right PPC's role in spatial representation is limited, and firmly establishes that this structure does not play a fundamental role in the visual perception of egocentric distance. Egocentric distance perception may be subserved by structures that are more diffusely distributed in the brain; one possibility, suggested by the lesions that typically underlie visual disorientation, is that bilateral structures are involved $[12,14]$.

A second clear finding is that the parietal group performed normally in all our tasks requiring spatial updating during walking. This indicates that the right PPC is not critically involved in updating self-location along straight paths. We found this surprising, considering the aggregation of evidence for impaired eye and arm position updating after PPC lesions $[15,16,29,65]$. Before abandoning the notion that the PPC is a critical structure for updating during locomotion, however, other types of locomotion and more complex trajectories should be tested. Specifically, the role of the PPC in updating during whole-body rotations has not been conclusively established; updating during rotations could conceivably be impaired even though updating along linear paths remains intact. Consistent with this view, several studies implicating the PPC in performing mental transformations of the body in space (an activity potentially related to self-motion updating) employed rotations as their critical manipulation [6,57,67]. The Locomotor Maze test also involves whole-body rotations, and has been found to elicit deficits in PPC patients [55]. Preliminary work in our laboratory suggests that although some patients with PPC lesions are indeed impaired at sensing their motion during passive whole-body rotations, others are not [43]. One way to reconcile these findings is to assume that different regions of the PPC are specialized for updating particular muscle groups and/or particular sensory systems. As mentioned in the Introduction, there is other evidence to support this notion. This important issue could be clarified in future work by using within-subjects designs to test PPC-injured individuals in updating tasks that systematically target a variety of specific muscle groups.

Our results leave open several possibilities concerning the potential neural substrates of self-location updating. Left hemisphere PPC patients have not yet been tested on these tasks, so it is possible that the left PPC is the critical structure. Several investigators have proposed that distinct neural circuits underlie the processing of spatial regions very near the body (peripersonal space) versus more distant regions (extrapersonal space) $[8,46,52]$. In particular, Previc [46] has proposed that the right hemisphere plays a greater role in peripersonal operations, with the left hemisphere more specialized for extrapersonal operations. This framework provides a good account of the apparently greater incidence of deficits in eye position updating after right PPC damage [29], and leads to the strong prediction that updating during walking should be more disturbed after left PPC lesions. Future testing with left hemisphere patients promises to shed light on this issue. Evidence is scant for lateralization of the neural substrates of arm position updating, but there are reports that lesions to or stimulation of the left PPC can lead to deficits in updating during reaches $[15,65]$, at least for reaches made by the contralateral arm.

Alternatively, some structure other than the PPC may be crucial for self-location monitoring. The fact that patient JM did show remarkably specific deficits in updating supports this view, particularly because these deficits arose after lesions confined to the right hemisphere. This suggests, at least, that this function is not subserved by a highly distributed, bilateral network of brain structures. In rats, lesions to brain regions in and around the hippocampal formation have been shown to impair path integration performance (for a review, see [48]), although as yet no single structure has been identified as critical $[1,48,61]$. In addition, a small proportion of cells in the monkey hippocampus have been shown to respond to linear and/ or rotational whole-body motion [41]. This region does not appear to be damaged in JM. He does, however, have extensive lesions in the right thalamus, particularly the anterior portion. The anterior thalamic nuclei in rats are thought to play a crucial part in updating head orientation [60,61], but in humans its relevance for updating along linear paths is unknown. The homology of the circuitry and functional architecture between rats and humans is far from established, and although JM's injuries include regions that have been associated with some forms of updating, he also has extensive lesions elsewhere. Therefore, we remain cautious about further speculation on the causal link between his brain lesions and his updating deficits.

Another possibility is that the PPC is involved in updating self-location during walking, but the walking tasks we used were not sufficiently sensitive to detect its influence. One reason this might be true is that our visually-directed walking task was performed without vision and took several seconds to execute. There is evidence that the PPC is fundamental for the control of visually-guided behaviors, while a distinct neural stream (running from primary visual cortex to terminate in the temporal lobe) controls actions conducted without visual guidance [40]. In general, when a delay is imposed between the occlusion of a visual target and the onset of an action directed to that target, there are distinct changes in the accuracy and kinematics of the action, consistent with the hypothesized influence of the temporal processing stream $[25,53]$. The amount of delay required to recruit involvement 
of the temporal stream depends to some extent upon the scale of the action: small scale behaviors such as eye movements show evidence of temporal stream influence after very short latencies (e.g., less than 500 ms) $[22,66]$, whereas larger scale behaviors such as reaching do not begin to show the influence until $2 \mathrm{~s}$ or more $[25,53]$. Because visually-directed walking is a relatively large scale behavior, the required delay could be sufficiently long that walks of several seconds continue to be controlled by the PPC despite the absence of visual guidance. The fact that our parietal patients were not impaired at walking to previously-viewed targets, however, suggests that this is not the case. In this view, updating during the walk could have been controlled by the temporal stream of visual processing, which presumably remained intact in these patients. Interestingly, the three patients in our brain-injured control group who had occipito-temporal lesions also performed the walking tasks normally. Although some version of the notion of functional specialization in the dorsal and ventral streams may be able to account for our data, a more definitive answer will await future testing.

JM could walk accurately and precisely to previously-viewed targets, and yet was quite imprecise at estimating self-motion of approximately the same extent when the walk was guided by the experimenter. The precision of responding also dropped significantly for the other participants in the study, although to a lesser degree. What differences between the two tasks best accounts for these performance decrements? Good performance in walking to previously-viewed targets rules out general deficits in spatial memory. One possible distinction is that in goal-directed walking, target locations may be represented in a more concrete fashion than are distances specified through time via locomotion, which by contrast must be synthesized through a more abstract process. Any task requiring spatial updating that is not performed in the service of goal-directed action may have a negative impact on response precision by virtue of this abstraction process. We consider this possibility to be unlikely, however. One task that requires such an abstraction is indicating the egocentric distance of a target by walking an equivalent distance in another direction instead of directly to the target. This abstraction does not affect the overall accuracy or precision of healthy humans, even when they turn 90 or $180^{\circ}$ before pacing off an equivalent distance $[18,56]$. Thus, abstraction processes involved in performing non-goal-directed walking do not provide a good account of why our participants were less precise when indicating distances presented by experimenter-guided locomotion. Although it is possible that JMs much larger drop in precision is due to such an abstraction process, we feel this is unlikely because he was able to perform the abstractions necessary to verbally estimate distances and to choose matching distances after a delay.

Another way of characterizing the differences between the two tasks is that in visually-directed walking, the observer actively produces locomotion toward the target, whereas when the stimulus distance is specified by blindfolded locomotion, the extent of walking is controlled by the experimenter. Strictly speaking, walking is not passively controlled in the latter case, because just as in visually-directed walking, the observer actively generates the muscular commands to produce locomotion; only the extent of locomotion is determined by the experimenter. Thus, as the walk begins, the observer has virtually no knowledge about the magnitude of the upcoming trajectory. During visually-directed walking, on the other hand, vision of the target provides the observer with knowledge of the extent of the walking path before updating begins. This prior knowledge could have an important facilitory effect upon updating. One possibility is that this information is used to generate a neural representation of the predicted extent of self-motion (i.e., a goal state for the path integrator) [65]. During the walk, the selfmotion that is sensed on-line can then be compared with the predicted value. An error signal such as this may prove to play a central role in facilitating self-location updating. We hope to pursue this important issue in future testing of our patient JM, whose responses showed a strong dissociation on updating tasks with and without a specified target.

\section{Acknowledgements}

This work was supported by NIH grant F32 MH11791 and JSMF grant 97-17 to JWP and NIH MH54246 to MB. A preliminary report of this work was presented in November, 1998 at the annual meeting of the Psychonomics Society in Dallas, TX. We thank Jack Loomis, Carl Olson, Carol Colby and two anonymous reviewers for helpful comments on earlier versions of this paper. We also thank Catherine Ewell for assistance in conducting the experiment, Peter Gao for help in preparing the imaging scans, the Pat Arato Aphasia Center for the use of its facilities, and Anthony Wingen and Kira Barbour for scheduling assistance.

\section{References}

[1] Alyan S, McNaughton BL. Hippocampectomized rats are capable of homing by path integration. Behavioral Neuroscience 1999;113:19-31.

[2] Andersen RA, Essick GK, Siegel RM. Encoding of spatial location by posterior parietal neurons. Science 1985;230:456-8.

[3] Behrmann M, Nelson J, Sekuler E. Visual complexity in letter- 
by-letter reading: "Pure" alexia is not so pure. Neuropsychologia 1998;36:1115-32.

[4] Bisiach E, Pattini P, Rusconi ML, Ricci R, Bernardini B. Unilateral neglect and space constancy during passive locomotion. Cortex 1997;33:313-22.

[5] Bisiach E, Vallar G. Hemineglect in humans. In: Boller F, Grafman J, editors. Handbook of neuropsychology, vol. 1. Amsterdam: Elsevier, 1988. p. 195-222.

[6] Bonda E, Petrides M, Frey S, Evans A. Neural correlates of mental transformations of the body-in-space. Proceedings of the National Academy of Sciences USA 1995;92:11,180-4.

[7] Böök A, Gärling T. Maintenance of orientation during locomotion in unfamiliar environments. Journal of Experimental Psychology: Human Perception \& Performance 1981;7:9951006.

[8] Brain WR. Visual disorientation with special reference to lesions of the right cerebral hemisphere. Brain 1941;64:244-72.

[9] Carey DP, Dijkerman HC, Milner AD. Perception and action in depth. Consciousness and Cognition 1998;7:438-53.

[10] Colby CL, Goldberg ME. Space and attention in parietal cortex. Annual Review of Neuroscience 1999;22:319-49.

[11] Corlett J. The role of vision in the planning and guidance of locomotion through the environment. In: Proteau L, Elliott D, editors. Vision and motor control. Elsevier, 1992. p. 375-97.

[12] Critchley M. The parietal lobes. London: Edward Arnold, 1953.

[13] Da Silva JA. Scales for perceived egocentric distance in a large open field: comparison of three psychophysical methods. American Journal of Psychology 1985;98:119-44.

[14] De Renzi E. Disorders of space exploration and cognition. New York: Wiley, 1982.

[15] Desmurget M, Epstein CM, Turner RS, Prablanc C, Alexander GE, Grafton ST. Role of the posterior parietal cortex in updating reaching movements to a visual target. Nature Neuroscience 1999;2:563-7.

[16] Duhamel JR, Goldberg ME, Fitzgibbon EJ, Sirigu A, Grafman J. Saccadic dysmetria in a patient with a right frontoparietal lesion: the importance of corollary discharge for accurate spatial behaviour. Brain 1992;115:1387-402.

[17] Elliott D. The influence of walking speed and prior practice on locomotor distance estimation. Journal of Motor Behavior 1987; 19:476-85.

[18] Farrell MJ, Thomson JA. On-line updating of spatial information during locomotion without vision. Journal of Motor Behavior 1999;31:39-53.

[19] Faugier-Grimaud S, Frenois C, Stein DG. Effects of posterior parietal lesions on visually guided behavior in monkeys. Neuropsychologia 1978;16:151-68.

[20] Gallistel CR. The organization of learning. Cambridge, MA: MIT Press, 1990.

[21] Gauthier I, Behrmann M, Tarr MJ. Can face recognition really be dissociated from object recognition? Journal of Cognitive Neuroscience 1999;11:349-70.

[22] Gnadt JW, Bracewell RM, Andersen RA. Sensorimotor transformation during eye movements to remembered visual targets. Vision Research 1991;31:693-715.

[23] Gogel WC, Loomis JM, Newman NJ, Sharkey TJ. Agreement between indirect measures of perceived distance. Perception \& Psychophysics 1985;37:17-27.

[24] Goldberg ME, Colby CL, Duhamel J-R. Representation of visuomotor space in the parietal lobe of the monkey. Cold Spring Harbor Symposia on Quantitative Biology 1990;55:72939.

[25] Goodale MA, Jakobson LS, Keillor JM. Differences in the visual control of pantomimed and natural grasping movements. Neuropsychologia 1994;32:1159-78.

[26] Hallett PE, Lightstone AD. Saccadic eye movements to flashed targets. Vision Research 1976;16:107-14.
[27] Hartje W, Ettlinger G. Reaching in light and dark after unilateral posterior parietal ablations in the monkey. Cortex 1973;9:346-54.

[28] Hécaen H, Tzortzis C, Masure MC. Spatial orientation difficulties in a route-finding test by patients with unilateral cortical lesions. Perception 1972;1:325-30.

[29] Heide W, Blankenburg M, Zimmermann E, Kompf D. Cortical control of double-step saccades: implications for spatial orientation. Annals of Neurology 1995;38:739-48.

[30] Holmes G. Disturbances of visual orientation. British Journal of Ophthalmology 1918;2:449-68.

[31] Holmes G, Horrax G. Disturbances of spatial orientation and visual attention with loss of stereoscopic vision. Archives of Neurological Psychiatry 1919;1:385-407.

[32] Jakobson LS, Archibald YM, Carey DP, Goodale MA. A kinematic analysis of reaching and grasping movements in a patient recovering from optic ataxia. Neuropsychologia 1991;29:803-9.

[33] Klatzky RL, Loomis JM, Golledge RG, Cicinelli JG, Doherty $\mathrm{S}$, Pellegrino JW. Acquisition of route and survey knowledge in the absence of vision. Journal of Motor Behavior 1990;22:19 43.

[34] Loomis JM, Da Silva JA, Fujita N, Fukusima SS. Visual space perception and visually directed action. Journal of Experimental Psychology: Human Perception \& Performance 1992;18:906-21.

[35] Loomis JM, Da Silva JA, Philbeck JW, Fukusima SS. Visual perception of location and distance. Current Directions in Psychological Science 1996;5:72-7.

[36] Loomis JM, Klatzky RL, Golledge RG, Cicinelli JG, Pellegrino JW, Fry PA. Nonvisual navigation by blind and sighted: assessment of path integration ability. Journal of Experimental Psychology: General 1993;122:73-91.

[37] Loomis JM, Klatzky RL, Golledge RG, Philbeck JW. Human navigation by path integration. In: Golledge RG, editor. Wayfinding behavior: cognitive mapping and other spatial processes. Baltimore, MD: Johns Hopkins Press, 1999. p. 125-51.

[38] Mapelli D, Behrmann M. The role of color in object recognition: evidence from visual agnosia. Neurocase 1997;3:237-47.

[39] Maurer R, Séguinot V. What is modelling for? A critical review of the models of path integration. Journal Of Theoretical Biology 1995;175:457-75.

[40] Milner AD, Goodale MA. The visual brain in action. Oxford, England: Oxford University Press, 1995.

[41] O'Mara SM, Rolls ET, Berthoz A, Kesner RP. Neurons responding to whole-body motion in the primate hippocampus. Journal of Neuroscience 1994;14:6511-23.

[42] Perenin M-T, Vighetto A. Optic ataxia: a specific disruption in visuomotor mechanisms. I. Different aspects of the deficit in reaching for objects. Brain 1988;111:643-74.

[43] Philbeck JW, Behrmann M, Loomis JM. Spatial updating during self-rotations after right posterior parietal lesions. Abstracts of the Psychonomics Society 1999;4(94).

[44] Philbeck JW, Loomis JM. Comparison of two indicators of perceived egocentric distance under full-cue and reduced-cue conditions. Journal of Experimental Psychology: Human Perception \& Performance 1997;23:72-85.

[45] Philbeck JW, Loomis JM, Beall AC. Visually perceived location is an invariant in the control of action. Perception \& Psychophysics 1997;59:601-12.

[46] Previc FH. The neuropsychology of 3-D space. Psychological Bulletin 1998;124:123-64.

[47] Ratcliff G. Disturbances of spatial orientation associated with cerebral lesions. In: Potegal M, editor. Spatial abilities: development and physiological foundations. New York: Academic Press, 1982. p. 301-31.

[48] Redish AD. Beyond the cognitive map: from place cells to episodic memory. Cambridge, MA: MIT Press, 1999. 
[49] Riddoch G. Visual disorientation in homonymous half-fields. Brain 1935;58:376-82.

[50] Rieser JJ. Dynamic spatial orientation and the coupling of representation and action. In: Golledge RG, editor. Wayfinding behavior: cognitive mapping and other spatial processes. Baltimore, MD: Johns Hopkins University Press, 1999. p. 16890.

[51] Rieser JJ, Ashmead DH, Talor CR, Youngquist GA. Visual perception and the guidance of locomotion without vision to previously seen targets. Perception 1990;19:675-89.

[52] Rizzolatti G, Matelli M, Pavesi G. Deficits in attention and movement following the removal of postarcuate (area 6) and prearcuate (area 8) cortex in macaque monkeys. Brain 1983;106:655-73.

[53] Rossetti Y. Implicit short-lived motor representations of space in brain damaged and healthy subjects. Consciousness and Cognition 1998;7:520-58.

[54] Schwartz M. Haptic perception of the distance walked while blindfolded. Journal of Experimental Psychology: Human Perception and Performance 1999;25:852-65.

[55] Semmes J, Weinstein S, Ghent L, Teuber H-L. Spatial orientation in man after cerebral injury: I. Analyses by locus of lesion. Journal of Psychology 1955;39:227-44.

[56] Sinai MJ, Ooi TL, He ZJ. Terrain influences the accurate judgement of distance. Nature 1998;395:497-500.

[57] Sirigu A, Duhamel J-R, Cohen L, Pillon B, Dubois B, Agid Y. The mental representation of hand movements after parietal cortex damage. Science 1996;273:1564-8.
[58] Steenhuis RE, Goodale MA. The effects of time and distance on accuracy of target-directed locomotion: does an accurate short-term memory for spatial location exist? Journal of Motor Behavior 1988;20:399-415.

[59] Stein JF. The representation of egocentric space in posterior parietal cortex. Behavioral and Brain Sciences 1992;15:691-700.

[60] Taube JS. Head direction cells recorded in the anterior thalamic nuclei of freely moving rats. Journal of Neuroscience 1995;15:70-86.

[61] Taube JS, Goodridge JP, Golob EJ, Dudchenko PA, Stackman RW. Processing the head direction cell signal: a review and commentary. Brain Research Bulletin 1996;40:477-86.

[62] Thomson JA. Is continuous visual monitoring necessary in visually guided locomotion? Journal of Experimental Psychology: Human Perception \& Performance 1983;9:427-43.

[63] Vecera SP, Behrmann M. Spatial attention does not require preattentive grouping. Neuropsychology 1997;11:30-43.

[64] Wilson B, Cockburn J, Halligan P. Behavioral inattention test. Flempton, Bury St. Edmunds, Suffolk, England: Thames Valley Test Company, 1987.

[65] Wolpert DM, Goodbody SJ, Husain M. Maintaining internal representations: the role of the human superior parietal lobe. Nature Neuroscience 1998;1:529-33.

[66] Wong E, Mack A. Saccadic programming and perceived location. Acta Psychologica 1981;48:123-31.

[67] Zacks J, Rypma B, Gabrieli JDE, Tversky B, Glover GH. Imagined transformations of bodies: an fMRI investigation. Neuropsychologia 1999;37:1029-40. 